\title{
Erratum to: Do newly marketed generic medicines expand markets using descriptive time series analysis and mixed logit models? Korea as an exemplar and its implications
}

Hye-Young Kwon ${ }^{1}$ and Brian Godman ${ }^{2,3^{*}}$

\section{Erratum}

Following the publication of this article [1], it was brought to our attention that Fig. 2 contains an error: the legends for graphs $\mathrm{b}$ and $\mathrm{c}$ are interchanged.

The corrected Fig. 2 is provided below:

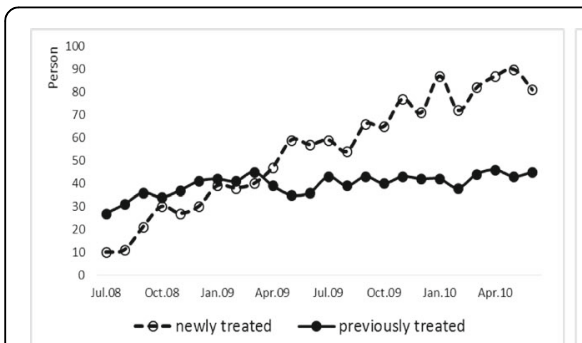

a) No. of patients on generic atorvastatin

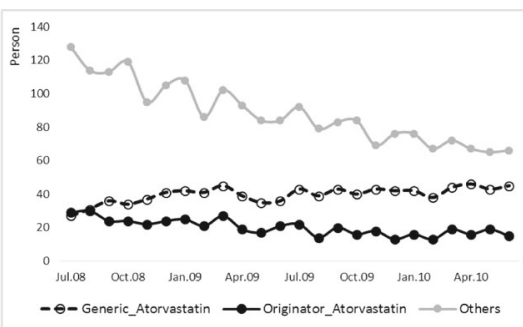

b) No. of patient in the previously treated group

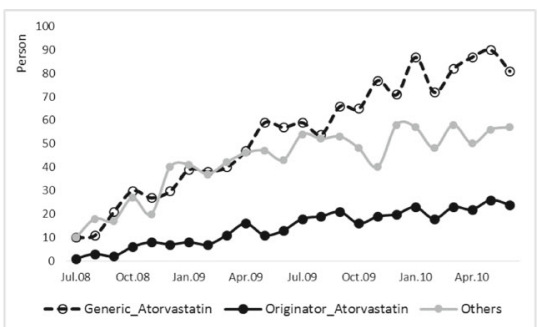

C) No. of patient in the newly treated group

Note : Others include simvastatin, lovastatin, pravastatin, fluvastatin, rosuvastatin and pitavastatin

Fig. 2 Number of patients prescribed overtime

\section{Author details}

'Division of Biomedicine \& Public Health, Mokwon University, Daejeon 35349, South Korea. ${ }^{2}$ Division of Clinical Pharmacology, Department of Laboratory Medicine, Karolinska Institute, Karolinska University Hospital Huddinge, Stockholm SE-141 86, Sweden. ${ }^{3}$ Strathclyde Institute of Pharmacy and Biomedical Sciences, University of Strathclyde, Glasgow G4 ORE, UK.
Received: 23 February 2017 Accepted: 23 February 2017

Published online: 15 March 2017

\section{Reference}

1. Kwon H-Y, Godman B. Do newly marketed generic medicines expand markets using descriptive time series analysis and mixed logit models? Korea as an exemplar and its implications. BMC Health Serv Res. 2016;16:130. doi:10.1186/s12913-016-1356-z.

\footnotetext{
* Correspondence: Brian.Godman@ki.se

${ }^{2}$ Division of Clinical Pharmacology, Department of Laboratory Medicine, Karolinska Institute, Karolinska University Hospital Huddinge, Stockholm SE-141 86, Sweden

${ }^{3}$ Strathclyde Institute of Pharmacy and Biomedical Sciences, University of Strathclyde, Glasgow G4 ORE, UK
} 